\title{
Interactive Direct Volume Rendering of Dural Arteriovenous Fistulae in MR-CISS Data
}

\author{
C. Rezk-Salama ${ }^{1}$, P. Hastreiter ${ }^{1}$, K. Eberhardt ${ }^{2}$, B. Tomandl ${ }^{2}$, and T. Ertl ${ }^{1}$ \\ 1 Computer Graphics Group, Department of Computer Science \\ University of Erlangen-Nuremberg \\ Am Weichselgarten 9, 91058 Erlangen, Germany \\ \{rezk|hastreiter|ertl\}@informatik. uni-erlangen.de \\ 2 Division of Neuroradiology, Department of Neurosurgery \\ University of Erlangen-Nuremberg \\ Schwabachanlage 6, 91054 Erlangen, Germany
}

\begin{abstract}
Dural arteriovenous fistulae are the cause of various somatic diseases. For their analysis DSA is used in medical practice. However, the injection of contrast dye into the vertebral segmental arteries is a time-consuming and highly difficult procedure. Aiming at a reduction of the number of injections required for the detection and exact localization of the pathology, we introduce a new approach which provides meaningful visualization of MR-CISS volumes. Due to the limited spatial resolution of the image data an explicit segmentation of the small vascular structures is extremely difficult. Therefore, we propose a fast sequence of filtering operations and volume growing in order to separate the areas of background, spinal cord and cerebrospinal fluid. Consecutively, we suggest implicit segmentation within these sub-volumes. This is achieved by interactively adjusting transfer functions of pre-defined color lookup tables with a few manipulation operations. Thereby, the tiny target vessels contained within the area of cerebrospinal fluid are clearly delineated. Overall, the presented non-invasive approach ensures an optimal spatial understanding of vessel structures in relation to the surrounding anatomy and contributes significantly to reduce the number of injections during DSA examinations.
\end{abstract}

Keywords: Direct Volume Rendering, MR-CISS, Analysis of Dural Arteriovenous Fistulae, Surgery Planning

\section{Introduction}

Dural arteriovenous fistulae ( $d A V F)$ are pathologic connections between arterious and venous blood vessels within the vertebral column [1]. Such malformations cause a variety of somatic diseases, ranging from back pain to paraplegia and physical disabilities. Possible treatments are coagulation of the pathologic structure or excision of the whole abnormal area during a neurosurgical intervention.

The comprehensive analysis of dAVFs requires detailed knowledge of the related vascular structures in order to localize the malformation. Digital subtraction angiography ( $D S A)$ is still the method of choice to obtain a clear delineation 
of the vessels within the spinal column. However, a contrast medium has to be injected to differentiate the vessels from the surrounding tissue. This leads to projection images which contain no depth information. In order to determine the exact location of a fistula, multiple injections into the vertebral segmental arteries on both sides are necessary which is a time-consuming process. In order to accelerate the analysis and to reduce the risk of injuries to the spinal cord, it is desirable to minimize the number of injections.

With magnetic resonance $(M R)$ it is possible to scan the vessels within the spinal column non-invasively, if a CISS (Constructive Interference in the Steady State) sequence [2] is applied. Revealing high contrast between the vascular structures and the cerebrospinal fluid (CSF) the slice images allow to substitute myelography since all the necessary information is available [3]. However, their two-dimensional (2D) representation is insufficient to track the course of the tiny vessels. This is mandatory to determine the location of a fistula allowing to perform DSA examinations more precisely and thereby to reduce the number of injections considerably. Therefore, an appropriate three-dimensional (3D) visualization of the MR-CISS data is required. This contributes considerably to understand the spatial relation of the involved structures producing meaningful images of spinal vascular structures with a diameter which is often below $1 \mathrm{~mm}$.

After a short overview in section 2 explaining the representation of the target structures within MR-CISS slice images, the applied visualization technique based on 3D-texture mapping is discussed in section 3. Time-consuming explicit segmentation of the target vessels is avoided by using implicit delineation. This is conveniently obtained with transfer functions which are manipulated interactively to adjust pre--defined lookup tables for color an opacity values. As an advantage only a coarse separation of CSF and the spinal cord is required which is easily achieved with a sequence of fast and simple pre-processing steps, presented in section 4 . Consecutively, section 5 describes the pre-defined color lookup tables and the interactive process to adjust the transfer functions to a specific data set. Finally, section 6 presents several clinical examples demonstrating the value of the suggested approach.

\section{MR-CISS Data}

The flexibility of magnet resonance imaging (MRI) allows to differentiate a great variety of tissues non-invasively by using different sets of scanning parameters. The MR-CISS sequence provides image data with high signal of CSF and fat tissue, whereas vascular structures have low intensity. As can be seen in figure 1 the area between the spinal cord and the dura is filled with CSF which contains the target vessels. Further-on, the dura is surrounded by bone structures of low intensity and partly by epidural fat of high signal values. Since the spinal cord as well as the vascular and the bony structures are in the same range of data values it is impossible to apply a simple maximum intensity projection (MIP). Contrary to that the explicit segmentation of the tiny vessels is a difficult and error-prone task since the resolution of the vessels is very limited and partial 
volume effects occur. However, using direct volume rendering semi-transparent views allow to delineate the vessels if an individual color lookup table is only locally applied to the area of CSF. An important prerequisite is the ability to manipulate the transfer functions interactively. This ensures accurate implicit segmentation within a very short time.

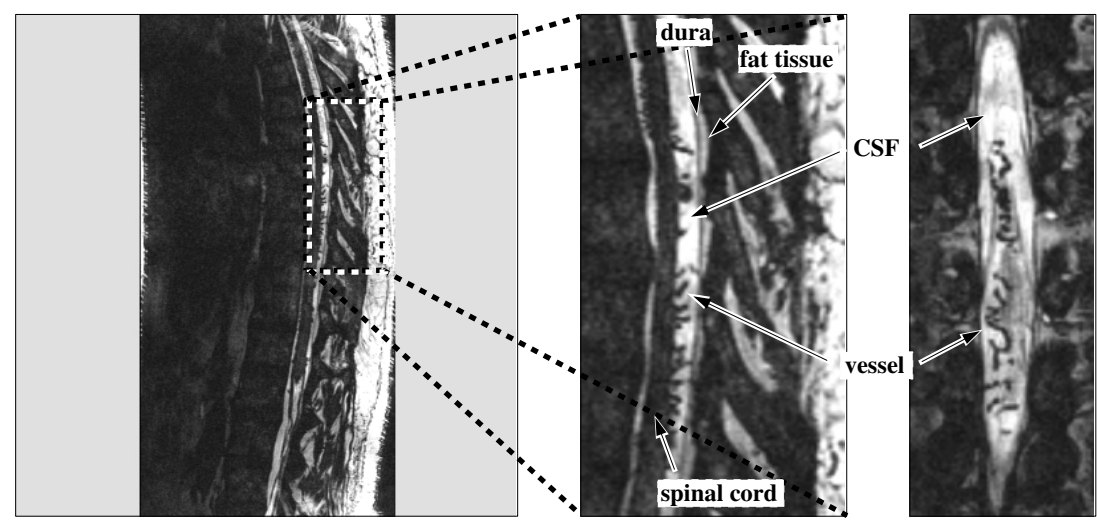

Fig. 1. MR-CISS with dAVF in the area of the thoracic spine: (left) Original saggital slice image — (middle) zoomed saggital view showing target vessels within CSF and (right) zoomed coronal view.

\section{Visualization}

In comparison to other approaches based on polygonal representations $[4,5]$, direct volume rendering has proved to be superior for the comprehensive and meaningful visualization of tomographic data [6,7]. Additionally, the combination of interactive manipulation and resulting images of high quality are indispensable for clinical application [8,9]. As presented previously [10] this is guaranteed with 3D texture mapping which provides hardware accelerated trilinear interpolation. According to the sampling theorem a $3 \mathrm{D}$ view of the volume is generated by drawing an adequate number of equidistant polygons parallel to the image plane with respect to the current viewing direction ("volume slicing"). During rasterization the respective polygons are textured with their corresponding image information directly obtained from the 3D-texture by trilinear interpolation. The final image is produced by successive blending of the textured polygons back-to-front onto the image plane. Due to the hardware accelerated interpolation and blending operations, the time consumed for rendering is negligible compared to software solutions.

During the rasterization process, transfer functions are applied to the $3 \mathrm{D}-$ texture, that specify color and opacity for each voxel value of the original data. The hardware integration allows to modify the lookup tables interactively providing direct visual feedback. Thereby, semi-transparent views lead to a fast 
and convenient implicit segmentation of the image data. This is most useful in pathologic cases with tiny and complex target structures. Moreover, it is the best way to deal with partial volume effects or noise inherent in the data which are usually very difficult to cope with using explicit segmentation.

For the interactive assignment of transfer functions, there are specific texture color tables of considerable depth on high-end graphics computers. They allow to apply the concept of "tagged volumes" which was previously presented in [11]. Using a voxel-based segmentation of the data, a unique tag number is assigned to every voxel in order to divide the data into disjoint subsets. According to the number of tags, the global lookup table is split into partitions. Thereby, individual transfer functions for color and opacity values are available for every sub-volume allowing to manipulate the 3D-representation locally.

In contrast to the described strategy based on 3D-texture mapping, approaches are currently gaining attention which avoid the computationally expensive trilinear interpolation by using 2D-textures and a shear-warp factorization of the viewing matrix [12]. However, they are less applicable for a detailed visualization during the analysis of tiny structures. Due to a fixed number of sampling points along the rays of sight and the spatially insufficient bilinear interpolation the resulting images might contain visual artifacts or appear blurred. This makes the clear delineation of small structures extremely difficult, especially if they are zoomed closely for a detailed inspection.

\section{Segmentation}

According to section 2 the slice images of MR-CISS data show high contrast between CSF and the spinal vessels. This is caused by the low intensity values of the vascular information and the high signal of CSF. However, both the surrounding tissue and the spinal cord are represented in the same range of intensity values as the target vessels. This prohibits an implicit delineation of the vascular structures if a lookup table is used which affects the volume data globally. Therefore, it is our strategy to isolate the region of CSF including the vascular structures and to apply transfer functions only locally. This approach is much faster and more convenient than an explicit segmentation of the vessels which might easily miss important features.

Subsequently, the sequence of pre-processing steps is explained which is used to separate the CSF including the spinal vessels, the spinal cord and the remaining part of the image data defined as background:

1. In most cases noise reduction is necessary as a first step to optimize the data for further pre-processing. Regions of higher homogeneity are obtained by anisotropic diffusion [13] while the exact object boundaries are preserved.

2. In order to segment the region of the CSF, containing the vascular structures, a morphologic 3D grey-value closing operation with a spherical filter kernel is applied to the voxel data. This operation removes the dark vascular structures within the region of CSF of high intensity, such that the whole region can be easily extracted by a simple threshold operation. For optimal 
results the size of the spherical filter kernel must be greater than the largest vessel diameter, but smaller than the diameter of the spinal cord.

3. Successively, the closed region of CSF is extracted by volume growing. Using bounding boxes to prevent volume off-shoots, the segmentation is computed stepwise starting at the top of the vertebral column.

4. Although this first segmentation already contains all the interesting structures, for an enhanced spatial understanding of the resulting images it is useful to additionally obtain a segmentation of the spinal cord. Again, this is easily achieved with volume growing of the closed image data, using the previous segmentation as a boundary.

5. Based on the segmentation results, the original image data is attributed using unique tag numbers for the CSF, the spinal cord and the surrounding dark tissue (see figure 2). Due to the immediate visual control and the low computational expense of the processing steps, the presented user-guided sequence leads to fast and robust segmentation results.
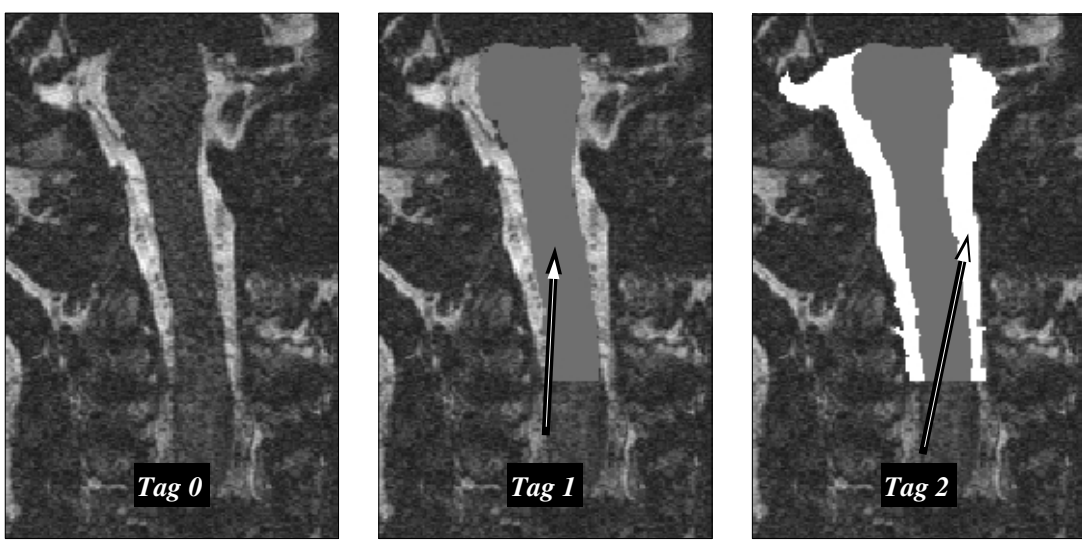

Fig. 2. Explicit segmentation of the significant regions represented by a tagged volume: Background (Tag 0), spinal cord (Tag 1), CSF including target vessels (Tag 2).

\section{$5 \quad$ Transfer Functions}

Using the coarse segmentation of the data described in the previous section, a detailed implicit delineation is obtained directly during volume rendering by interactively adjusting transfer functions. For every tagged sub-volume, four separate curves are used describing the correlation between the original data values and the displayed color components and opacity (RGBA).

Figure 3 shows the transfer functions which lead to the visualization presented in figure 5. As additional source of information the intensity histogram 
of the volume data is displayed within the diagram. For the background region (tag 0), the opacity is set to a constant low value and a linear ramp is specified for the color components. This results in a semi-transparent representation which supports the anatomical orientation. To reveal the small vessels within the CSF (tag 2), the setting of the opacity function causes an implicit segmentation. Starting with high opacity for the low data values of the vascular structures, a slope must be adjusted to render the CSF of high intensity transparently. The red component is adjusted with a decay towards higher data values to enhance the impression of depth. The green and blue color components are completely switched off for this tag. Finally, the transfer functions of the spinal cord ( tag 1) are set to full opacity and green color to additionally enhance the contrast.

To speed up the process of adjusting the transfer functions, pre-defined templates are used. These lookup tables look similar to those presented in figure 3 . During the analysis of the image data the respective functions are either manipulated individually or as a combined set after grouping them arbitrarily. In order to find the optimal representation of every subregion only a few and simple operations are necessary. These comprise vertical and horizontal translations or simple movements of a handle in order to change the angle of a linear mapping.
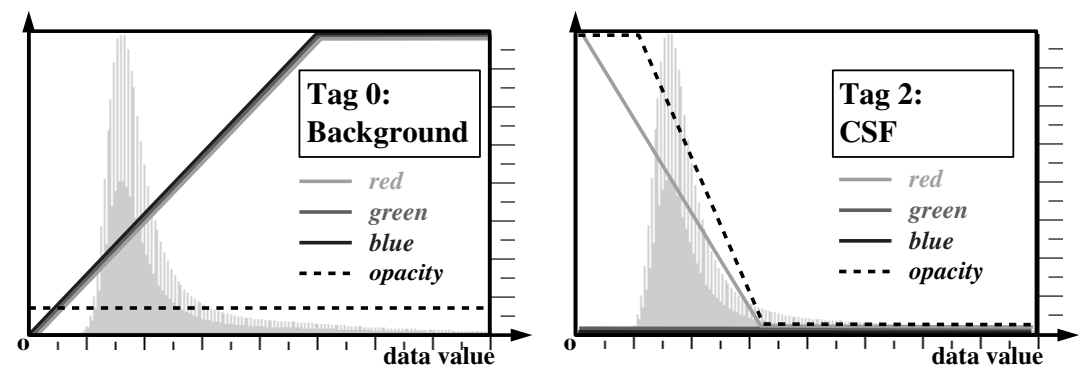

Fig. 3. Intensity histogram and transfer functions for the visualization shown in figure $5(e, f)$ : (left) Setting for the semi-transparent background region (right) Setting applied to delineate the vascular structures contained in the subvolume of the CSF.

\section{Results and Discussion}

The presented approach was so far applied to the data of 12 patients with different locations of dAVFs. All MR-CISS volumes were acquired with a Siemens MR Vision 1.5 Tesla scanner which provides the necessary resolution to resolve the tiny vessel structures appropriately. In all cases volumes were used consisting of images with a $512^{2}$ matrix and 40-70 slices. The size of the voxels was set to $0.5 \times 0.5 \times 0.7 \mathrm{~mm}^{3}$. In order to guarantee high frame rates the consecutive 3D-visualization was exclusively perform on a SGI Onyx2 (R10000, 195MHz) with BaseReality graphics hardware providing 64 Mbytes of texture memory. 
To our experience direct volume rendering is the only visualization technique to produce meaningful 3D-representations of dAVFs contained in MR-CISS data. The following four cases demonstrate the clinical value of our strategy:

- Figure 4 comprises a DSA image of a dAVF and the 3D-visualization of MR-CISS data. This example shows the excellent correspondence between DSA and the 3D representation. The fully opaque spinal cord in green color enhances the spatial understanding. Additionally, clip planes are used to display the surrounding bone structures for the anatomical orientation.

- A highly complex vessel structure close to the medulla oblongata is presented in figure 5. While the spatial orientation is extremely difficult using DSA projections, this example clearly demonstrates the benefit of a $3 \mathrm{D}$ representation for the localization of the nidus of the fistula.

- The example in figure 6 shows a dAVF in the area of the lumbar spine. Although the vascular structure is surrounded by the roots of spinal nerves, the malformation is clearly delineated. In this case, the nidus of the fistula is located outside the area of CSF and thus cannot be visualized.

- Figure 7 displays a detailed 3D representation of a complex venous vessel in the area of the thoracic spine. Due to epidural fat tissue, the closing operation lead to segmentation artifacts (partly visible in $(b)$ ), which are successfully removed by interactive adjustment of independent clip planes.

\section{- Note: the color images can be found at}

http://www9.informatik.uni-erlangen.de/eng/research/vis/dAVM/miccai.html

As demonstrated in these examples, the suggested approach based on noninvasively acquired image data assists tremendously to find the fistula and to define the related vertebrae. Finally, DSA is still applied since it represents a gold standard for the examination of dAVFs. However, knowing the exact location of the malformation the time-consuming process of multiple DSA projections is optimized by reducing the required number of injections to a minimum.

The size of the target structures and the spatial distribution of data values within MR-CISS data require a complex and time-consuming process to segment the vessels explicitly. Thus, for clinical application it is more appropriate to separate the whole area of CSF in a robust way. Overall the coarse pre-segmentation involving noise reduction, morphologic operations and volume growing takes approximately 10-15 minutes. For the meaningful and fast delineation of the vessel structures contained in the segmented subregion of the CSF the interactive adjustment of transfer functions is crucial, ensuring direct visual feedback. Using pre-defined color lookup tables, the adaptation of transfer functions to the individual data takes another 5 minutes. For interactive visualization 3D-texture mapping guarantees high frame rates and excellent image quality by using trilinear interpolation. 


\section{Conclusion}

An approach was suggested for the interactive 3D-visualization of dural arteriovenous fistulae. Using MR-CISS data only the CSF and the spinal cord have to be segmented explicitly. The meaningful delineation of the target vessels is then performed implicitly by adjusting pre-defined color lookup tables for each separated area. Direct volume rendering based on 3D-texture mapping ensures the required interactivity and the necessary image quality to analyze the tiny vessels. The presented results demonstrate the value of our approach in practice which proved to effectively reduce the number of injections required for DSA examination. Therefore, our method assists pre-operative planning as a valuable approach for patients with dural arteriovenous fistulae.

\section{References}

1. M. Hamilton, J. Anson, and R. Spetzler. The Practice of Neurosurgery, chapter Spinal Vascular Malformations, pages 2272-2292. Williams \& Wilkins, 1996. 42

2. M. Deimling and G. Laub. Book of Abstracts, chapter Constructive Interference in Steady State for Motion Sensitivity Reduction, page 842. Society of Magnetic Resonance in Medicine, 1989. 43

3. K. Eberhardt, I. Schäfer, M. Deimling, H.-P. Hollenbach, and F. Fellner. Diagnosis of Spinal Dural Arteriovenous Malformations Using a 3D-CISS Sequence. In Proc. of Soc. of Magn. Res. in Med., volume 2, 1997. 43

4. S. Nakajima, H. Atsumi, A. Bhalerao, F. Jolesz, R. Kikinis, T. Yoshimine, T. Moriarty, and P. Stieg. Computer-assisted Surgical Planning for Cerebrovascular Neurosurgery. Neurosurgery, 41:403-409, 1997. 44

5. M.Melgar, L. Zamorano, Z. Jiang, M. Guthikonda, V. Gordon, and F. Diaz. ThreeDimensional Magnetic Resonance Angiography in the Planning of Aneurysm Surgery. Comp. Aided Surgery, 2:11-23, 1997. 44

6. B. Kuszyk, D. Heath, D. Ney, D. Bluemke, B. Urban, T. Chambers, and E. Fishman. CT Angiography with Volume Rendering : Imaging Findings. American Jour. of Radiol. (AJR), pages 445-448, 1995. 44

7. G. Rubin, C. Beaulieu, V. Argiro, H. Ringl, A. Norbash, J. Feller, M. Dake, R. Jeffrey, and S. Napel. Perspective Volume Rendering of CT and MR Images: Applications for Endoscopic Viewing. Radiology, 199:321-330, 1996. 44

8. L. Serra, R. Kockro, C. Guan, N. Hern, E. Lee, Y. Lee, C. Chan, and W. Nowinsky. Multimodal Volume-Based Tumor Nerurosurgery Planning in the Virtual Workbench. In Proc. Med. Img. Comput. and Comp.-Assis. Interv. (MICCAI), volume 1496 of Lec. Notes in Comp. Sc., pages 1007-1015. Springer, 1998. 44

9. P. Hastreiter, C. Rezk-Salama, , B. Tomandl, K. Eberhardt, and T. Ertl. Fast Analysis of Intracranial Aneurysms based on Interactive Direct Volume Rendering and CT-Angiography. In Proc. Med. Img. Comput. and Comp.-Assis. Interv. (MICCAI), volume 1496 of Lec. Notes in Comp. Sc. Springer, 1998. 44

10. B. Cabral, N. Cam, and J. Foran. Accelerated Volume Rendering and Tomographic Reconstruction Using Texture Mapping Hardware. ACM Symp. on Vol. Vis., pages 91-98, 1994. 44 
11. P. Hastreiter, H. Çakmak, and T. Ertl. Intuitive and Interactive Manipulation of 3D Data Sets by Integrating Texture Mapping Based Volume Rendering into the OpenInventor Class Hierarchy. In Worksh. Bildverarb. f.d. Med. (BVM), pages 149-154, 1996. 45

12. P. Lacroute and M. Levoy. Fast Volume Rendering Using a Shear-Warp Factorization of the Viewing Transform . Comp. Graphics, 28(4):451-458, 1994. 45

13. G. Gerig, O. Kübler, R. Kikinis, and F. Jolesz. Nonlinear Anisotropic Filtering of MRI Data. IEEE Trans. on Med. Imag., 11(2):221-232, 1992. 45
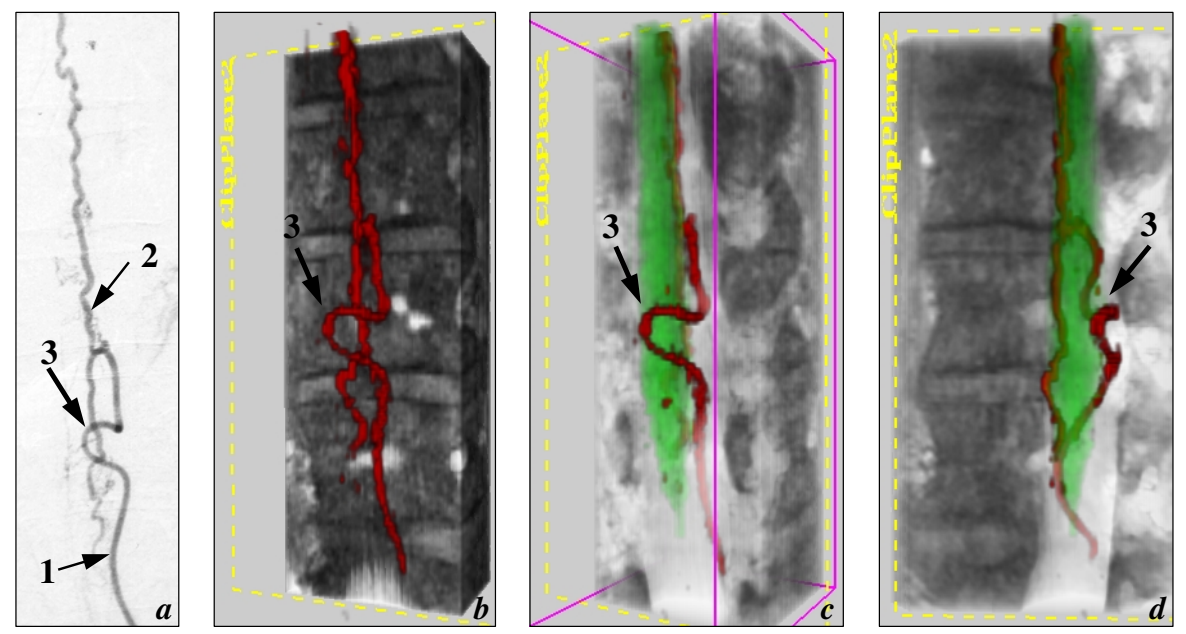

Fig. 4. Dural AV fistula in the area of the lower thoracic spine: (a) DSA shows the feeding artery $(1 \longrightarrow)$ and a perimedullary fistula $(2 \longrightarrow)-(b)$ Direct volume rending of $\mathrm{MR}-C I S S$ shows optimal correspondence $(3 \longrightarrow)$ with the DSA - $(c, d)$ The integration of the surrounding anatomy conveys the relation to the spinal cord (green) and the bone structures of the vertebral column. 

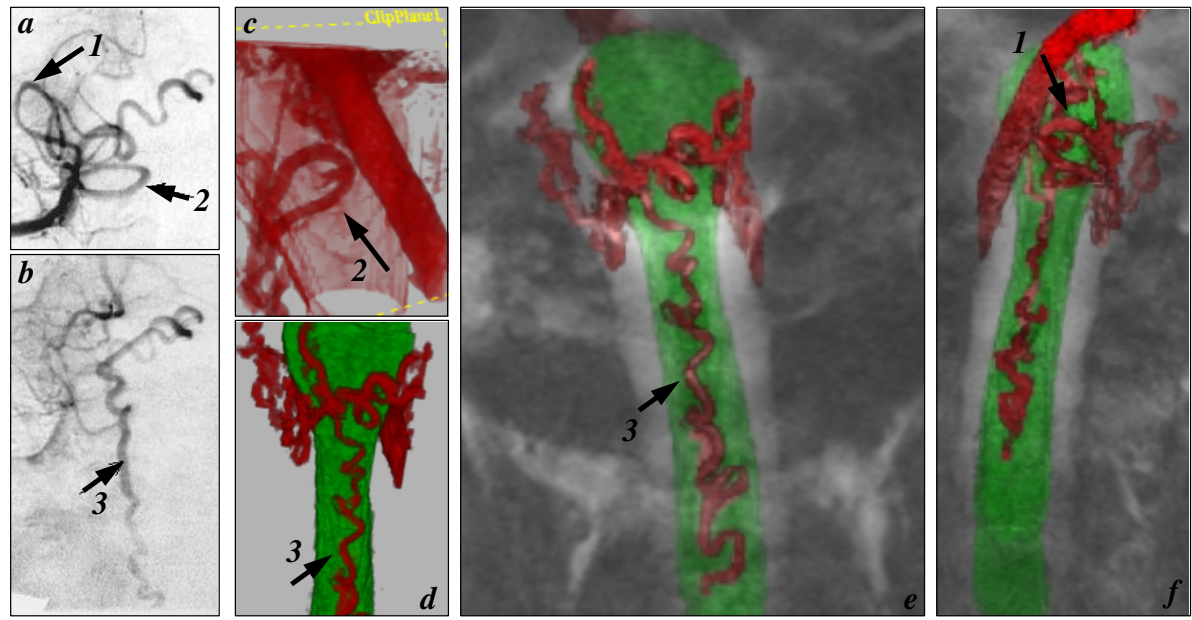

Fig. 5. Dural AV fistula in the area of the medulla oblongata: $(a, b)$ DSA showing the vascular malformation - $(c-f)$ Direct volume rendering leads to an excellent delineation showing the semi-transparent CSF and the opaque target vessels (c), the opaque vessels and the opaque spinal cord (d), and the relation to the surrounding anatomy $(e, f)$.
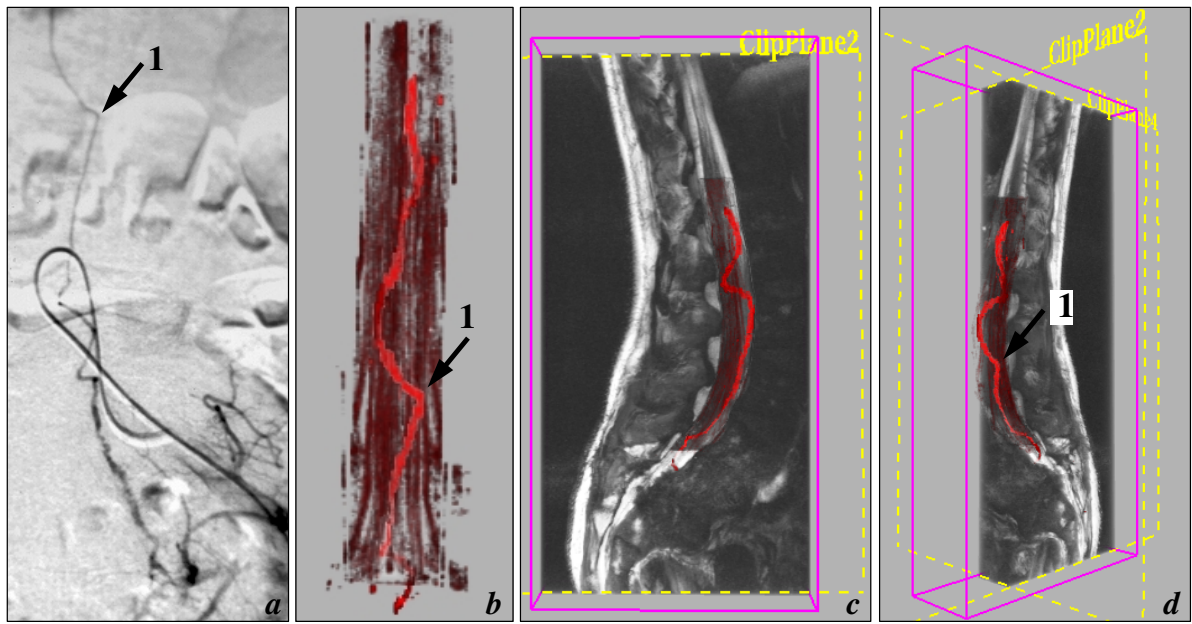

Fig. 6. Dural AV fistula in the area of the lumbar spine: (a) DSA shows a fistula $(1 \longrightarrow)$ coming from the right internal iliac artery. - (b) Volume rendering of the fistula and the roots of spinal nerves. $(c, d)$ Lateral and frontal view of the malformation in relation to the surrounding anatomy. 

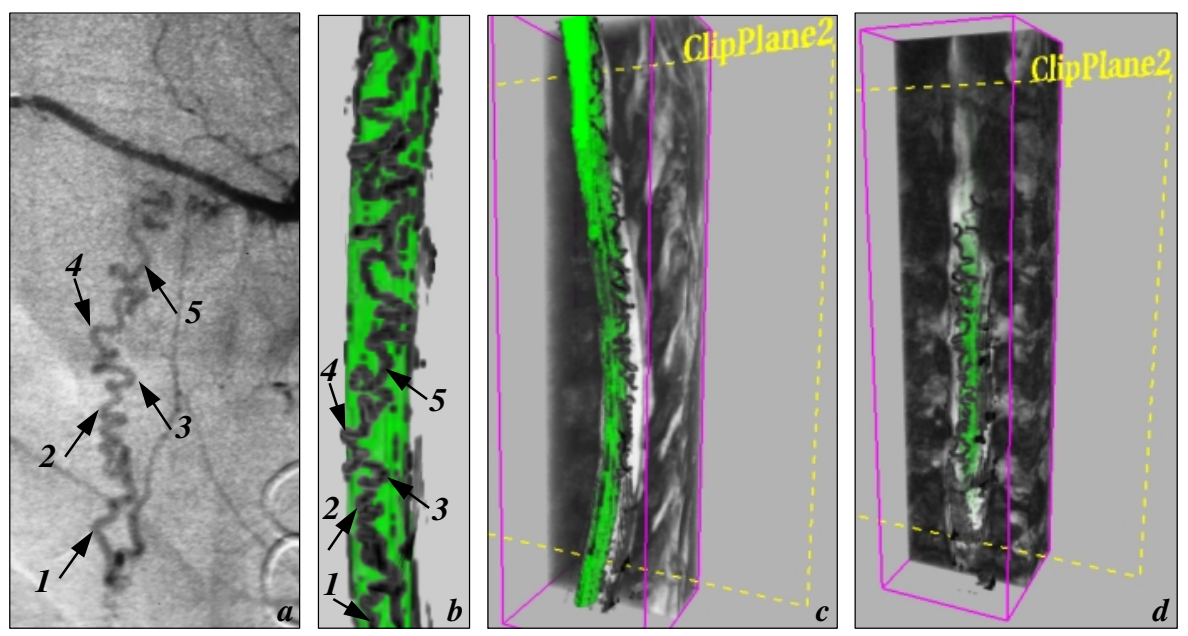

Fig. 7. Dural AV fistula in the area of the thoracic spine: Comparison of the complex vessel structure in a DSA projection image (a) and a corresponding $3 \mathrm{D}$ visualization of a $\mathrm{MR}-C I S S$ volume (b) including a lateral (c) and a dorsal view $(d)$. 